\title{
Design of Implanted RFID Tags for Passive Sensing of Human Body: The STENTag
}

\author{
Cecilia Occhiuzzi, Member, IEEE, Giordano Contri, and Gaetano Marrocco, Member, IEEE
}

\begin{abstract}
Numerical processing of passive UHF-RFID tags' response may provide physical insight about the hosting object or about the nearby environment. This idea is here extended to implanted antennas with the purpose to sense the evolution of some human physiological and pathological process involving a local change of effective permittivity inside the body. The goal is to understand how master the design of this class of devices taking into account both communication and sensing capabilities. An ad hoc design methodology is here presented and discussed by means of a realistic medical case concerning the modification of an endo-vascular device to achieve a STENTag able to sense the state of the vessel wherein it has been implanted.
\end{abstract}

Index Terms-Biosensor, implantable biomedical device, passive sensing, RFID.

\section{INTRODUCTION}

B ESIDE the common applications to Logistics, the Radio Frequency Identification (RFID) technology has been recently recognized as potentially capable to produce additional information about the tagged object, such as its physical state and its time-evolution, without any specific embedded sensor or local power supply [1]. The physical rationale of this idea, for which the tag acts as a self-sensing device, lies in the dependence of the tag's input impedance and of its radar cross section on the physical and geometrical features of the tagged object or, more in general, on the close surrounding environment. The possibility to monitor processes in evolution discloses interesting opportunities in telemedicine and human health monitoring in general, especially concerning implantable devices. One or more battery-less RFID radio-sensors could be integrated into implanted medical devices such as prosthesis, sutures, endovascular devices and orthopedic fixings, and then interrogated by an external reader (Fig. 1). By data acquisition at different times (days or even hours), the change history of geometrical or chemical features of the tissues could be collected, thus observing in an indirect way the healing process and possible complications, e.g., abnormal cell proliferation, edema and inflammatory events.

In general, the critical issues in the design of implanted radios are the feasibility of a reasonable link range [2], the low power

Manuscript received April 08, 2011; revised December 31, 2011; accepted February 25, 2012. Date of publication May 07, 2012; date of current version July $02,2012$.

The author are with the DISP-University of Roma Tor Vergata, Via del Politecnico, 1, 00133 Roma, Italy (e-mail: occhiuzzi@disp.uniroma2.it; marrocco@disp.uniroma2.it).

Color versions of one or more of the figures in this paper are available online at http://ieeexplore.ieee.org.

Digital Object Identifier 10.1109/TAP.2012.2198189

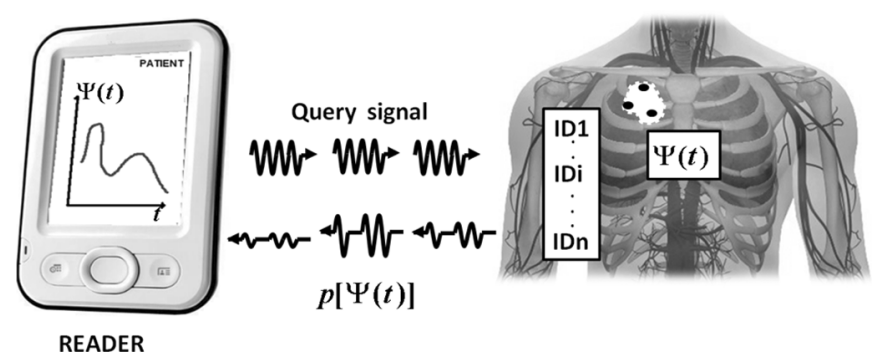

Fig. 1. The change of the electromagnetic response $p$ of implanted RFID tags may be related to a biologic process in evolution $\Psi(t)$ inside the human body.

consumption [3], biocompatibility [4] and miniaturization [5]. Implanted RFID tags were used in the past for tagging animals. Passive devices for human identification purposes have instead been proposed very recently in [6], as well as implantable antennas suitable to host biological sensors (blood-pressure [7], temperature, glucose). Patch-like antennas [2] and monopolelike antennas [8] have been studied for subcutaneous implants, while first examples of ingestible RFID capsules interrogated by a wearable reader can be found in [9].

Up to now, no attempt has been reported, according to our knowledge, to extract sensing data from passive RFID implanted sensor-less tags in addition to more traditional identification codes. Beside the critical issues shared by conventional RFID and implanted antennas, the very big challenge is the control of the sensitivity and of the dynamic range of the tag-as-sensor during the design task in addition to the usual communication requirements. Biological processes are characterized by a great variety of dynamic ranges in terms of evolution timeline and amplitude [10]-[14]. Some pathologies may show very slow and moderate evolutions, while in other cases, especially during acute events, rapid and large tissues modifications may be observed: the measurable response of RFID radio sensor needs therefore to be properly correlated to the process under observation. In any case, the problem is even more challenging due to the high permittivity and high losses of living tissues. As proved in [1], the electrical parameters of an RFID tag (impedance and gain) in these conditions are expected to be very little sensitive to the variation of the dielectric properties, because of the smoothing effect induced by the losses.

This paper proposes a complete procedure for the design of self-sensing implanted tags, here described with specific focus to the monitoring of biological ducts hosting a stent implant. A stent is a metal-mesh tubular device used to recover a stenosis [15], an abnormal narrowing in a blood vessel or in other tubular 
organ or structure, e.g., the occlusion of an artery due to abnormal accumulation of atherosclerotic plaque. The stent is surgically implanted into the vessel, then expands, keeps the vessel open thus improving blood flow, and stays in its seat permanently. Unfortunately, this procedure is not definitive since a new accumulation of tissue inside and around the stent may give rise to risk of in-stent restenosis (ISR). A common post-surgery requirement is thus monitoring the device status, or in other words the sensing of its local environment over time.

Starting from the basic equations governing the RFID sensing, it will be here shown how to choose the IC family, and in particular how to match the implanted antenna in order to maximize its sensitivity to the variation of the physical parameter under observation. Finally, by the help of a laboratory phantom, the design procedure is fully experimented over a stent prototype, the STENTag, in both fixed-and multi-frequency modalities.

\section{RFID-SEnSing EQuATIONS AND Design StRATEGIES}

We denote with $\Psi(t)$ a local physical or geometrical parameter of the body region which has to be monitored by the RFID platform. Specifically, $\Psi$ will be a shape factor of the biological process or, more generally, the local effective permittivity "sensed" by the tag's antenna.

The two-way reader-tag link [16] for UHF-RFID systems (overall World-wide band: $866-956 \mathrm{MHz}$ ) is commonly characterized under the far field assumption by using the Friis formula for the direct path, and the radar cross-section for the reverse one. Although the electromagnetic interaction with the human body is much more complicated, the free-space formalism is nevertheless here considered with the purpose to simplify the design methodology described next. The reliability of this choice will be discussed in Section III by the help of an accurate numerical simulation involving a realistic body phantom.

The conventional RFID formulas are hence rewritten making explicit the dependence on the variation of local parameters with the purpose to obtain some sensing metrics which can be derived by the reader's measurements. The power collected by the IC (1) and the power backscattered by the tag toward the reader (2) and collected by it, are

$$
\begin{aligned}
& P_{R \rightarrow T}(\Psi)=\left(\frac{\lambda_{0}}{4 \pi d}\right)^{2} P_{\mathrm{in}} G_{R}(\theta, \phi) G_{T}(\theta, \phi, \Psi) \tau(\Psi) \eta_{p} \\
& P_{R \leftarrow T}(\Psi)=\frac{1}{4 \pi}\left(\frac{\lambda_{0}}{4 \pi d^{2}}\right)^{2} P_{\mathrm{in}} G_{R}^{2}(\theta, \phi) \operatorname{rcs}_{T}(\theta, \phi, \Psi) \eta_{p}
\end{aligned}
$$

where $d$ is the reader-tag distance, $G_{R}(\theta, \phi)$ is the gain of the reader antenna, $G_{T}(\theta, \phi, \Psi)$ is the gain of the tag's antenna when placed into the body which hence includes all the absorption and obstruction phenomena in the inside-to-outside (and converse) propagation. $P_{\text {in }}$ is the power entering the reader's antenna, $\eta_{p}$ is the polarization mismatch between the reader and the tag, $\tau(\Psi)$ is the power transmission coefficient of the tag

$$
\tau[\Psi]=\frac{4 R_{\text {chip }} R_{A}(\Psi)}{\left|Z_{\text {chip }}+Z_{A}(\Psi)\right|^{2}}
$$

with $Z_{\text {chip }}=R_{\text {chip }}+j X_{\text {chip }}$ and $Z_{A}=R_{A}+j X_{A}$ input impedance of the RFID IC and of the antenna, respectively. $\operatorname{rcs}_{T}$ is the tag's radar cross-section

$$
\operatorname{rcs}_{T}(\Psi)=\frac{\lambda_{0}^{2}}{4 \pi} G_{T}^{2}(\theta, \phi, \Psi)\left(\frac{2 R_{A}(\Psi)}{\left|Z_{\text {chip }}+Z_{A}(\Psi)\right|}\right)^{2} \eta_{p}
$$

The backscattered power $P_{R \leftarrow T}$, is strictly correlated to the physical variation of the local environment through the change of impedance and gain of the tag.

A second indicator which can be measured by the reader is the turn-on power $P_{\text {in }}^{t o}(\Psi)$, e.g., the minimum input power $P_{\text {in }}$ through the reader's antenna forcing the tag to respond. It can be derived from (1), by imposing $P_{R \rightarrow T}=P_{\text {chip }}, P_{\text {chip }}$ being the IC sensitivity provided by the manufacturer

$$
P_{\text {in }}^{t o}(\Psi)=\left(\frac{\lambda_{0}}{4 \pi d}\right)^{-2} \frac{P_{\text {chip }}}{G_{R}(\theta, \phi) \eta_{p} G_{T}(\theta, \phi, \Psi) \tau(\Psi)} .
$$

The presence of the multi-path, caused by the interaction with the environment, is not taken into consideration here because of the expected small reader-tag distances achievable within the allowed emitted power. It was demonstrated in [17] that for low-gain tags, as in case of implanted antennas, the free-space formula is still useful to predict the read region.

The two indicators in (2) and (5) may be used as data inversion curves $p(\Psi)$ between measured data and the evolution $\Psi$ of the process. It could be useful to normalize each indicator by its value in a particular reference state, say $\Psi_{0}$, for instance collected at the time of the tag placement into the body.

\section{A. Design Parameters and Strategies}

The idea of self-sensing tags implies that there is no decoupling from the operative and structural point of view, between antenna and sensor, more precisely the antenna is the sensor and the sensor is the antenna. The sensitivity and the dynamic range of the system are thus strictly connected to the antenna's features, in particular to its quality factor, and definitely to its bandwidth (related to the tag's realized gain $G_{T} \tau$ [18]). Due to the high water content, however, human tissues are characterized by high permittivity and significant losses, so that the implanted tag will exhibit a large bandwidth and hence a generally poor sensitivity to the change of the local environment.

There are at least three degrees of freedom available to master the sensing performances of such a class of devices. The first one is the shape of the antenna which is however mainly constrained to medical requirements so that only a modest geometrical change is allowed. The second degree of freedom is the IC family having a specific power threshold and input impedance. As reported in [20], tags matched to ICs with very high phase angle (the ratio $\mathrm{Q}$ between the imaginary and the real part of the input impedance) exhibit higher sensitivity to the variation of antenna impedance vs. the change of the tagged objects and therefore they are preferred for sensing activities. The last available option is the state $\Psi_{m}$ (real or virtual) of the biological process where forcing the antenna to have the best impedance matching to the RFID IC. In this condition $\Psi_{m}$ is referred to as matched state. As recently investigated in [1] the choice of the matched state affects the position of the peak of power transfer coefficient versus the dynamic range of the process so that it is 


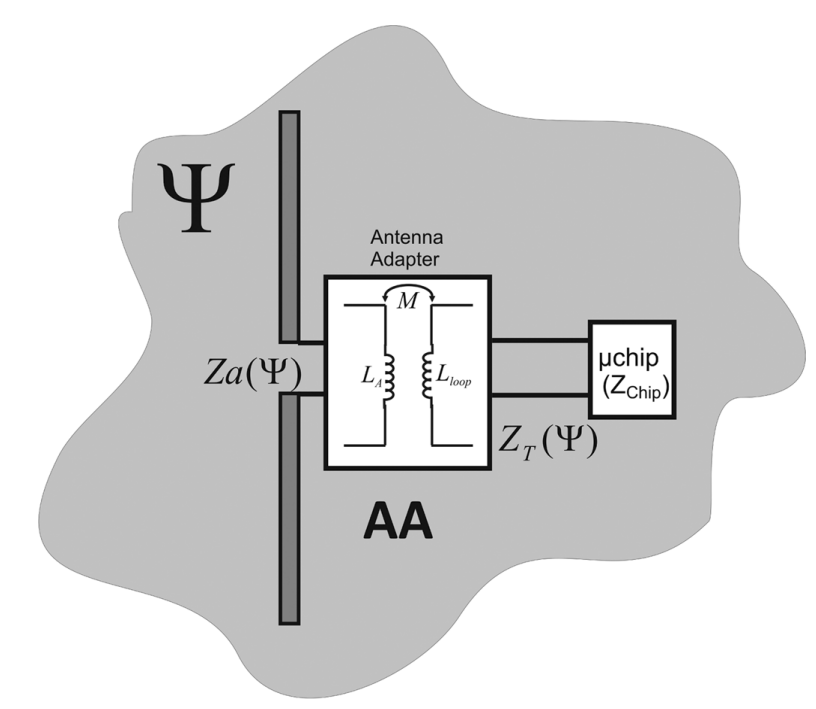

Fig. 2. Reference model to investigate the key issues in the design of implanted tags. Given a radiating structure, an antenna adapter is used to match such an antenna to a specific IC in a particular state $\Psi_{m}$ (the matched state) of the process to be observed.

possible to shift, and hence to localize, the curve of any sensing indicator vs. the change of the process parameter. By means of such a shaping of the data inversion curve it is expected to be able to emphasize the antenna response at the worsening of the pathology or instead to amplify the sign of healing.

The design parameters are here correlated together according to the reference model in Fig. 2 where the tag's antenna is connected to the IC via an Antenna Adapter $A A_{m}$. This one virtually applies to the antenna impedance $Z_{A}$ and yields a transformed impedance $Z_{T}$ which is matched to the specific IC such to get the best power transfer in the particular state $\Psi_{m}$ of the process (the matched state). The key parameters of the selfsensing tag are hence inter-correlated by the following matching equation:

$$
Z_{T}\left[S_{k}, A A_{m}\right]\left(\Psi_{m}\right)=Z_{\text {chip }, n}^{*}
$$

In (6) the asterisk denotes the complex conjugate, the $[. .$. indicates the design conditions and (...) the specific state of the process where the antenna's impedance is evaluated.

The effect of the antenna adapter could be obtained [18], for instance, by an inductively coupled loop placed in the close proximity of the radiating structure, a T-match or, more simply, by adding discrete inductors/capacitors, as shown later on in the experimental Section.

Following the previous considerations, it is possible to define basic guidelines to engineer the data inversion curves according to the specific process under monitoring, for instance to emphasize the variation of the early or late state of the process, as required case by case.

Denoting with $R$ the set of $\Psi$ states describing the evolution of the process under monitoring, it is required that the inversion curve (say $p(\Psi)$ in general) is monotonic in $R$ and with a given overall variation $s$, e.g.,

$$
\Delta p(R) \rightarrow s
$$

Moreover the radio-sensor has to be subjected to constraints over the maximum interrogation power $P_{\text {EIRP }}^{t o}=P_{\text {in }}^{t o} G_{R}$ in order to permit a suitable transcutaneous communication, at a given body-reader distance, for the whole process's evolution $R$ and in agreement with power regulations $\left(P_{\mathrm{Max}}\right)$ or with the expected capabilities of the reader

$$
P_{\text {EIRP }}^{\text {to }}(\Psi) \leq P_{\text {Max }}, \quad \forall \Psi \in R
$$

The shaping of the radio-sensor's response is mainly reduced to an iterative search for the most appropriate IC and mostly for the status $\Psi_{m}$ of the process where maximizing the power collected by the IC. The design procedure is coded as follows:

i) select the antenna shape $S_{k}$ together with the topology of the antenna adapter (a T-match, a coupled loop, or even discrete inductors/capacitors). These choices will be subjected to the specific medical constraints, the implantation locus and the available space over an existing implantable device, if any;

ii) select the most suitable IC family $\left(Z_{\text {chip }}\right)$ with the highest impedance phase angle Q;

iii) select a guess value for the matched state $\Psi_{m}$, belonging or not to the process evolution $R$. For instance the first guess for $\Psi_{m}$ could be that corresponding to the healthy condition or instead to the severe hilliness;

iv) calculate the corresponding input impedance $Z_{A}\left(\Psi_{m}\right)$;

v) estimate from inversion of (6) the parameters $A A_{m}$ of the antenna adapter so that the transformed impedance $Z_{T}$ of the antenna appears matched to the IC;

vi) calculate the turn-on power and the backscattered power for all $\Psi \in R$;

vii) verify the sensing and communication constraints in (7) and (8);

viii) if such conditions are matched then the parameters of the antenna adapter found in $v$ ) will give the final design;

ix) if the check in vii) is instead unsuccessful, then modify the matched state $\Psi_{m}$ and/or select a different IC and jump back to step iv).

The tag response to the process' changes (described by the impedance and the gain) needs to be estimated by measurements over phantoms, or even better by application of numerical electromagnetic models.

More in general, even the shape $S_{k}$ of the antenna, as well as the topology of the antenna adapter, could be introduced in the iterative loop of the design, with many more degrees of freedom but at the cost of highly increased computational complexity.

The design procedure is now illustrated with reference to a realistic medical case, e.g., the design of the STENTag, able to sense the healthy condition of an artery.

\section{THE STENT $_{\mathrm{AG}}$}

The in-stent restenosis (Fig. 3) could begin just after the implant of the stent into a body duct, due to hyper proliferation of neointimal cells (similar to muscular tissue) [13], as well as in a longer period due to the accumulation of new tissue, such as atherosclerotic plaques (similar to a mixture of water and fatty tissue) [14]. From a clinical and a prognostic point of view, the ISR can be usefully classified according to its evolution pattern 


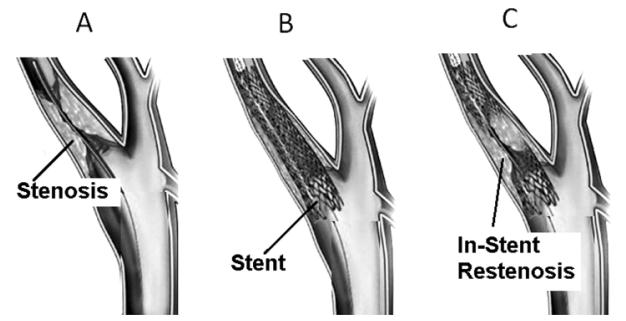

Fig. 3. Stenosis, stenting procedures and in-stent restenosis of a biological duct. Adapted from [22].

and grade. A possible site of stenting and hence of ISR is the carotid. As reported in [23], a more that 40\% diameter reduction of the vessel can be considered critical for the safety of the patient, while a reduction of more than $80 \%$ must be regarded extremely dangerous and requires a further surgical treatment. The most severe forms if IRS occur when the lesions are wide ( $>10 \mathrm{~mm}$ long) and extend beyond the margins of the stents. This latter form is referred to as diffuse proliferative ISR.

Since a vascular stent is typically fabricated with biocompatible metallic alloys, for instance the Nickel-Titanium (Nitinol) [24] with nice conducting features, some researchers have recently proposed to use the stent itself as a radiating elements to set-up a transcutaneous wireless telemetry system [25] where the status of the vessel is detected by a dedicated sensor integrated on board the stent. The idea to use the stent as sensor of restenosis has been instead investigated in [26], by relating the presence of cell proliferation and tissue growth to a low frequency $(0.1 \mathrm{~Hz}$ to $10 \mathrm{MHz})$ impedance measurement. However, the wired nature of the device (being necessary catheters to measure the impedance of the stent), makes the described platform not suited to a wireless telemetry system.

The stent is instead a natural candidate to achieve a selfsensing implanted RFID tag which would combine both mechanical and sensing capabilities. This new device, hereafter denoted as "STENTag", has been obtained from an existing stent after minimal geometrical modification and by the inclusion of an RFID IC.

The design guidelines in Section II, are applied to a commercial $L=4.3 \mathrm{~cm}$ long self-expanding stent [27]. The Antenna Adapter is here reduced to just an inductor connected in series to the chip, and hence the transformed impedance of the STENTag will be simply

$$
Z_{T}=Z_{A}+j \omega L_{m}
$$

The considered IC is the NXP-G2X having power sensitivity $P_{\text {chip }}=-15 \mathrm{dBm}$ and impedance at $870 \mathrm{MHz} Z_{\text {chip }}=15-$ $j 135 \Omega$ (corresponding to impedance phase angle $Q=9$ ). The $\mathrm{IC}$ is integrated into the device by means of an additional $l=1$ $\mathrm{cm}$ long Nitinol straight wire, protruding from the tubular grid (Fig. 8). The resulting object may be regarded as an asymmetrical dipole, with a hollow branch. Although a real implant of this augmented stent should require additional work, aimed to properly shape the added Nitinol wire and to preserve the biocompatibility, nevertheless this geometry is useful to demonstrate the design methodology and to understand the achievable sensitivity.
The process evolution $R$ to be monitored is the proliferation of tissue inside the stent, according to typical diffuse proliferative patterns: as the ISR increases, the healthy tissue (normally blood) is progressively replaced by neointima (muscle cells proliferation), up to stimulate, in the very long period, the possible formation of new atherosclerotic plaque. Because of all those tissues are characterized by different dielectric properties, the parameter $\Psi$ to be sensed is the equivalent complex permittivity $\bar{\epsilon}_{\text {stent }}$ inside the stent. The desired STENTag should have an overall variation of the power response of not less than 3 $\mathrm{dB}$ between the healthy condition and the full atherosclerotic restenosis, e.g.,

$$
\Delta p(R) \geq 3 d B=100 \%
$$

and a required turn-on power similar to the power emitted by hand-held smartphones

$$
P_{\text {in }}^{t o}(\Psi) \leq 0.25 W=24 \mathrm{dBm} \quad \forall \Psi \in R .
$$

\section{A. Model}

The application of the proposed methodology requires to set-up a numerical model of the neck and of the in-stent restenosis. Accurate digitalized body phantoms (one for all, the Visible Human [28]) are now available, and provide assessed databases of electromagnetic parameters of the organs. These models could be in principle used together with a Finite Difference Time Domain (FDTD) tool to predict the tag's response in presence of the restenosis' evolution. However, since laboratory experimentations have to be performed, and human or even animal implants are not feasible in this phase, a more simplified cylindrical model has been considered. It offers a reasonable control of the restenosis grade and, above all, it can be easily manufactured and experimented with high reproducibility.

The neck is hence simulated by cylinder of diameter $D=9$ $\mathrm{cm}$ and height $h=20 \mathrm{~cm}$ filled by a homogeneous dielectric having the weighted average permittivity of all the main tissues composing the neck, $\left(\bar{\epsilon}_{\text {neck }}=41.4-j 14.8\right)$, while a smaller offcentered $0.8 \mathrm{~cm}$-diameter cylinder simulates the human carotid and it is filled by blood-like dielectric $\left(\bar{\epsilon}_{\text {vessel }}\right)$. The sizes of the phantom have been chosen such to reproduce the overall characteristics of Visible Human model. In particular the height of the cylinder comprises also the bulk of the head and part of the shoulders.

The STENTag will be inserted inside the middle of the smaller cylinder, tight fitting its internal surface, in a localized volume simulating the diffuse proliferative restenosis. Such a district, of height $S=8 \mathrm{~cm}$, is homogeneously filled with a dielectric with variable permittivity $\left(\bar{\epsilon}_{\text {stent }}\right)$. It is worth noticing that, although the proposed model sensibly simplifies the ISR patterns, it could be assumed as a reasonable approximation of the phenomena, since the radial increase/decrease of the diffuse proliferative IRS can be converted into the change of an homogeneous dielectric having the weighted average permittivity of all the healthy and the unhealthy portions of the vessel. As specified in Table I seven conditions have been 
TABLE I

Re-Stenosis: Properties at 870 MHz of the Vessel's Dielectric

\begin{tabular}{|c|c|c|}
\hline Vessel's filler & $\begin{array}{l}\text { Theoretical Complex } \\
\text { Permittivity }\end{array}$ & $\begin{array}{l}\text { Measured Complex } \\
\text { Permittivity }\end{array}$ \\
\hline $\begin{array}{l}\text { 1- Healthy } \\
\text { vessel } \\
I S R=0 \%\end{array}$ & $\bar{\epsilon}_{1}=61.45-j 31.5$ & $\bar{\epsilon}_{1 m}=57.8-j 33.0$ \\
\hline $\begin{array}{l}\text { 2-Neointimal } \\
\text { proliferation } \\
I S R=50 \%\end{array}$ & $\bar{\epsilon}_{2}=58.22-j 25.3$ & $\bar{\epsilon}_{2 m}=56.17-j 27.6$ \\
\hline $\begin{array}{l}\text { 3-Neointimal } \\
\text { proliferation } \\
I S R=100 \%\end{array}$ & $\bar{\epsilon}_{3}=55-j 19.2$ & $\bar{\epsilon}_{3 m}=51.65-j 22.7$ \\
\hline $\begin{array}{l}\text { 4-Plaque } \\
\text { proliferation } \\
\text { grade } 1\end{array}$ & $\bar{\epsilon}_{4}=48.2-j 17.0$ & $\bar{\epsilon}_{4 m}=46.7-j 20.19$ \\
\hline $\begin{array}{l}\text { 5-Plaque } \\
\text { proliferation } \\
\text { grade } 2\end{array}$ & $\bar{\epsilon}_{5}=41.4-j 14.8$ & $\bar{\epsilon}_{5 m}=41.8-j 17.6$ \\
\hline $\begin{array}{l}\text { 6-Plaque } \\
\text { proliferation } \\
\text { grade } 3\end{array}$ & $\bar{\epsilon}_{6}=27.5-j 10.95$ & $\bar{\epsilon}_{6 m}=27.5-j 10.95$ \\
\hline $\begin{array}{l}\text { 7-Plaque } \\
\text { proliferation } \\
I S R=100 \%\end{array}$ & $\bar{\epsilon}_{7}=14.13-j 12.15$ & $\bar{\epsilon}_{7 m}=14.13-j 12.15$ \\
\hline
\end{tabular}
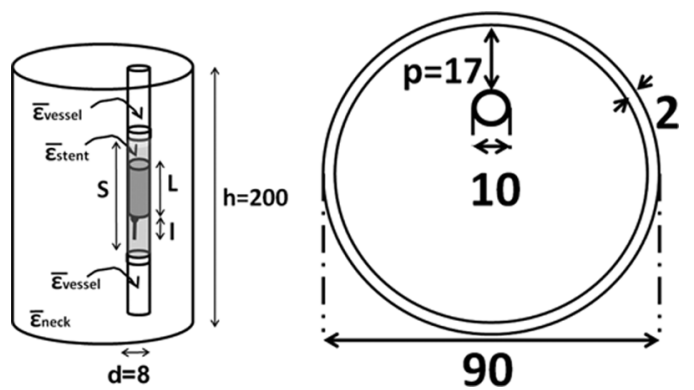

Fig. 4. Cylindrical model of the neck, the carotid and the ISR (side view and top view). The STENTag is inserted in the middle of the smaller cylinder, tightly fitting its internal surface, as in a real-life implant. The ISR is modeled has a localized (shadowed) volume with variable permittivity extending beyond the margins of the stents. Size in [mm].

simulated, starting from the blood, passing through the neointimal proliferation, down to a plaque restenosis. Finally, the meshed tubular part of the STENTag has been simulated as a continuous cylindrical surface as in [25].

The physical reliability of the considered cylindrical model has been preliminary validated in comparison with a more realistic anthropomorphic numerical phantom based on the Visible Human database, which includes a true description of the carotid and the surrounding tissues. At this purpose, a reference 2.5 cm dipole ( $\lambda / 2$ in the body), insulated by a $2 \mathrm{~mm}$-thick teflon coating, has been introduced within the two carotid models at corresponding positions (Fig. 5), assuming an healthy vessel, e.g., in absence of restenosis.

The results obtained by FDTD simulations are visible in Fig. 6 for what concerns the input impedance of the antenna and the electric field along the $\mathbf{x}$-line passing through the dipole's axis. The impedance estimations by the two models are quite in agreement with less than $2 \div 6 \Omega$ difference in the entire UHF (World-Wide) RFID Band. This is an expected result since the
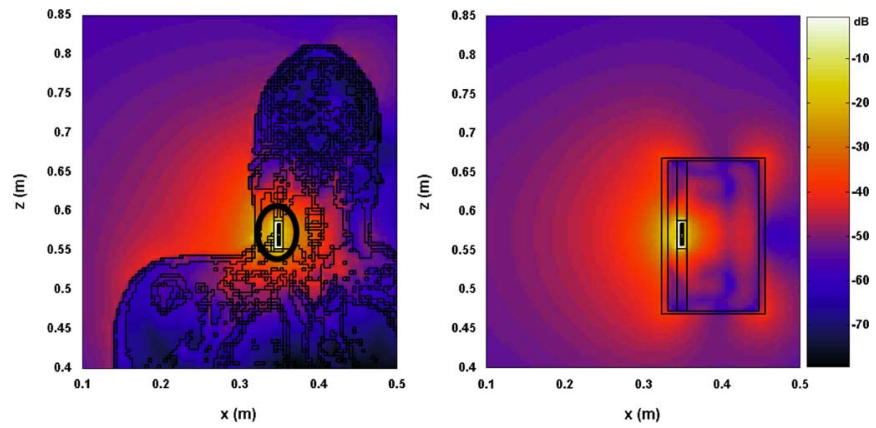

Fig. 5. Electromagnetic field ( $\mathrm{dB}$ scale) radiated by a $2 \mathrm{~mm}$-teflon-coated half-wave dipole implanted into the human carotid. Left) Frontal cross-section of Visible Human FDTD phantom; Right) Simplified cylindric phantom. The dipole is placed inside the carotid at position $x=34.8 \mathrm{~cm}$ and $z=56.8 \mathrm{~cm}$ in both the models.
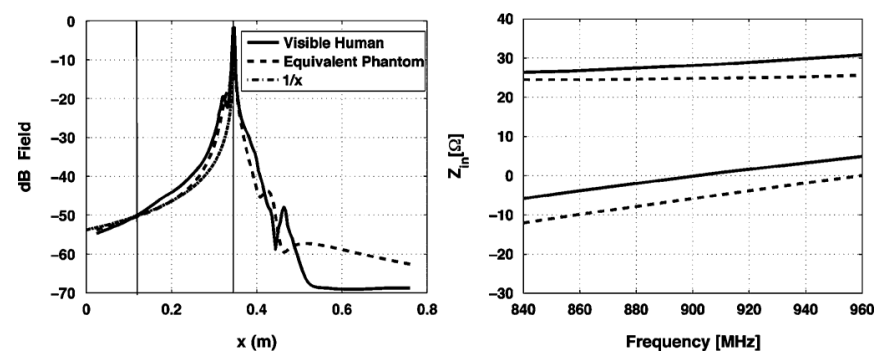

Fig. 6. (Left) Electric field along the $\mathrm{x}$-axis (at height $z=56.8 \mathrm{~cm}$ corresponding to the dipole's center) radiated by a half-wave dipole implanted in the human carotid for the two different numerical phantoms of Fig. 5. The typical far-field profile $1 / x$ has been also superimposed. (Right) Input impedance of the same dipole as predicted by the two numerical phantoms.

high losses of the human tissues force the interaction with the antenna to be very local. The cylindrical phantom is therefore adequate to capture the significant physics of the neck, at least in the considered frequency band.

Concerning the outside radiation, it can be observed that the field profiles are almost overlapped and well fitted by the $1 / x$ curve indicating the typical far-field attenuation. Therefore, just after a distance of about $15 \mathrm{~cm}$ from the locus of the implant, it is reasonable to assume the far-field approximation and hence to apply the equations in Section II to extract the RFID parameters of the STENTag, required for the design.

\section{B. Design Procedure}

Once fixed the antenna's shape, the topology of the antenna adapter, the RIFD IC, the design constraints and the models simulating the biological process under investigation, it is possible to iteratively apply the design procedure in Section II in order to search for the most suitable matched state $\Psi_{m}$ and the corresponding value of the inductance $L_{m}$. In all the considered cases the electromagnetic model is solved by an FDTD tool which directly provide the antenna gain and impedance, while the derived power functions in (2) and (5) are then obtained in post-processing assuming a reader-tag distance of 20 $\mathrm{cm}$ and a $5 \mathrm{~dB}$-gain reader's antenna in linear polarization. Fig. 7 shows the backscattered power as in (2) normalized to the turn on power $P_{\text {in }}^{t o}, p_{S}(\Psi)=P_{R \leftarrow T} / P_{\text {in }}^{t o}$ and the turn-on power as in (5) versus the ISR process for three possible matched states 

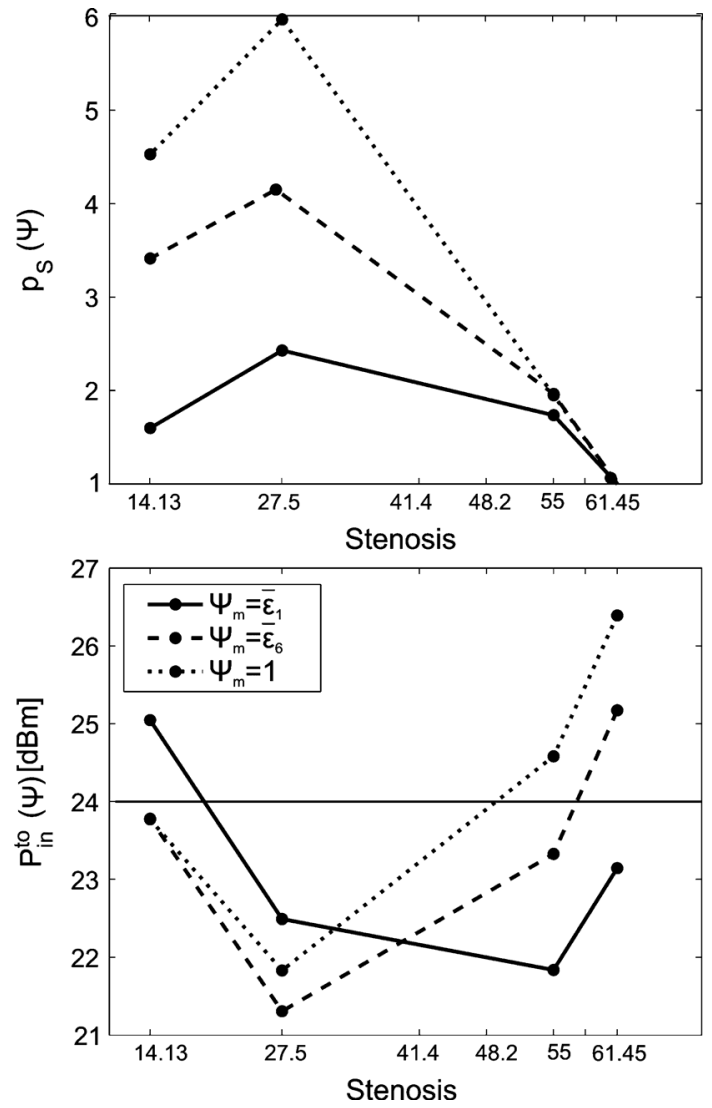

Fig. 7. Normalized backscattered power and turn-on power of the STENTag simulated at $870 \mathrm{MHz}$ during the ISR process for three different choices of the matched state. The simulated data are sorted according to the real part of the considered complex permittivity.

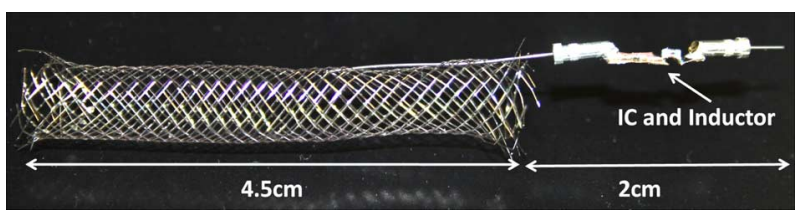

Fig. 8. STENTag prototype with a details of the integration of RFID IC and of the inductor.

$\Psi_{m}=\left\{\epsilon_{1}, \epsilon_{6}, 1\right\}$, calculated at the European UHF-RFID frequency $870 \mathrm{MHz}$.

Among the considered choices of $\Psi_{m}$, the case $\Psi_{m}=\bar{\epsilon}_{6}$ offers the best trade-off between sensing sensibility (the slope of the curves) and the communication reliability which is expected to improve since the turn-on power decreases as the vessel's state worsens. The simulated dynamic range of the backscattered power between the healthy stent and the atherosclerotic restenosis is about $1: 3$, while the maximum required turn-on power is $25 \mathrm{dBm}$, comparable with the power emitted by a mobile phone.

The resulting value of input impedance at $870 \mathrm{MHz}$ is $Z_{A}\left(\bar{\epsilon}_{6}\right)=29+j 22 \Omega$ and finally the matching inductor, as deduced from inversion of (6) through (9), is $L_{m}=30 \mathrm{nH}$.

\section{Prototypes and Experimentation}

A prototype of the STENTag is shown in Fig. 8. Since the Nitinol alloy can not be easily soldered, the wire components

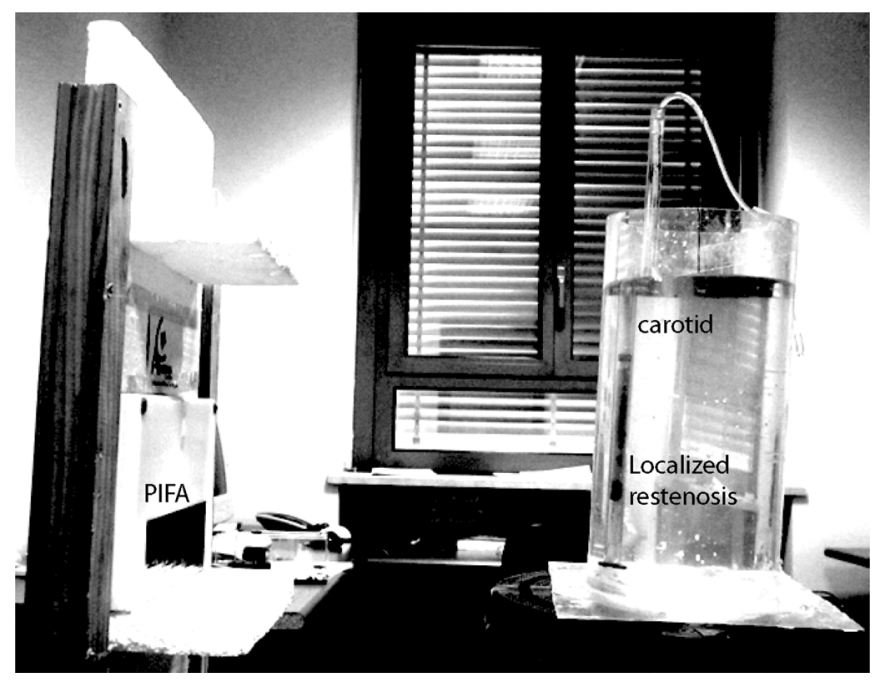

Fig. 9. Experimental set-up comprising the liquid phantom resembling the neck where the STENTag has been implanted, and the interrogation $5 \mathrm{~dB}$ patch antenna connected to the Thing-Magic M5e reader (not shown in the figure). The darker area in the central part of the phantom corresponds to the ISR. Such a volume is filled/emptied by means of a proper hydraulic circuit.

have been interconnected by means of crimped miniature connectors. Finally, the IC and the inductor have been insulated by a teflon tape (not shown in the figure) before the implant.

The STENTag has been experimented in vitro by means of equivalent liquid phantoms, according to the numerical model of Fig. 4. The larger and smaller cylinders have been fabricated by perspex pipes of thickness $2 \mathrm{~mm}$ and $1 \mathrm{~mm}$, respectively, and then filled with liquid mixtures of water, sugar and salt which simulates the dielectric properties of the human tissues according to the recipes in [29]. The measured permittivity of the liquid phantoms, averaged over 6 samples, are indicated in Table I and compared with the expected values from the recipes. The diffuse proliferative ISR has been rendered by dividing the small pipe into three parts. The central one, wherein the STENTag is placed, is dynamically filled/emptied by means of a proper hydraulic circuit (Fig. 9).

All the measurements have been performed within the UHF band (840-960 MHz) by means of a UHF Thing-Magic M5e reader, connected to a $5 \mathrm{~dB}$ gain linear polarized patch antenna (Fig. 9) which is placed at $20 \mathrm{~cm}$ from the neck phantom, as in the simulations. The reader permits to control the input power by $0.5 \mathrm{~dB}$ steps and the receiving module performs a 8 -bit digital conversion of the signals backscattered by the tag.

Fig. 10 shows the curves of the measured turn-on power in the UHF band, versus the change of permittivity of the liquid filling the STENTag. The curves have similar behavior but are clearly distinguishable. The power required to turn on the chip reduces as the stenosis's grade gets worse. A nearly $3 \mathrm{~dB}$ dynamic range is observed at each frequency when moving from the healthy condition to complete plaque occlusion of the duct.

A more quantitative representation including the error analysis and the comparison with the computer simulations at 870 $\mathrm{MHz}$ is given in Fig. 11 for both the backscattered power $p_{S}(\Psi)$ and the turn-on power $P_{\text {in }}^{\text {to }}(\Psi)$. The mean value and the standard deviation (the gray region) have been calculated starting from 


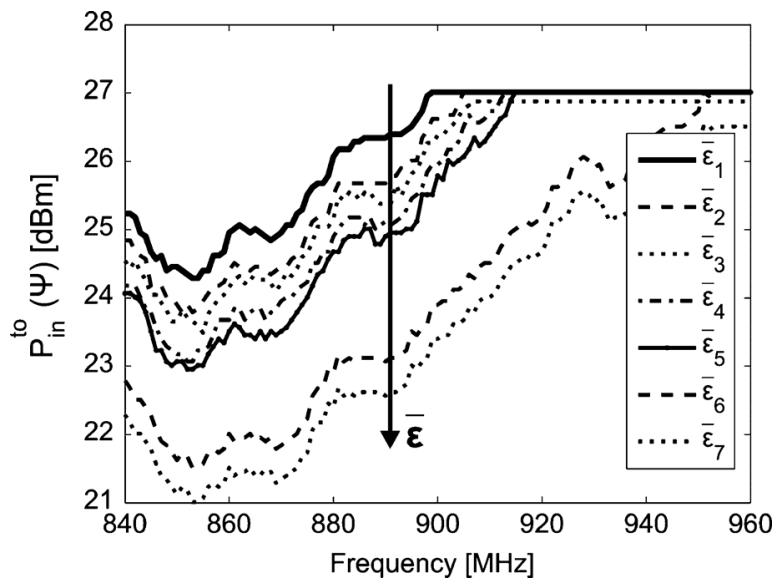

Fig. 10. Measured turn-on power in the UHF (World- Wide) Band for the STENTag prototype, versus the change of the liquid permittivity filling the carotid-like pipe.
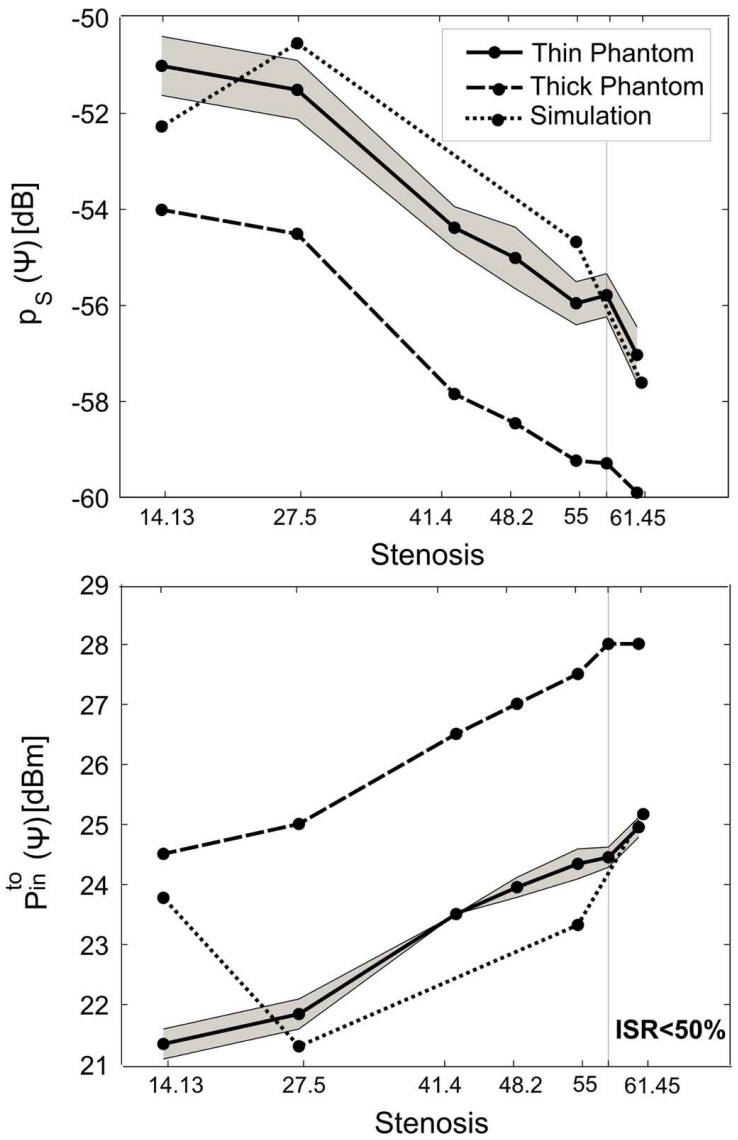

Fig. 11. Measured (mean and shaded uncertainty region) and simulated (a) backscattered power and (b) turn on power for the STENTag prototype at 870 $\mathrm{MHz}$, on varying the liquid composition inside the carotid-like phantom. The measured data are sorted according to the real part of the considered complex permittivity.

nine measurements at different angular offset (within $\pm 30^{\circ}$ ) between the reader's antenna and the tag.

First of all, it is apparent that in spite of the high tolerance in the fabrication of the tag and, non least, the typical instabilities of the receiving module of the RFID reader, simulations and measurements are in reasonable agreement, demonstrating
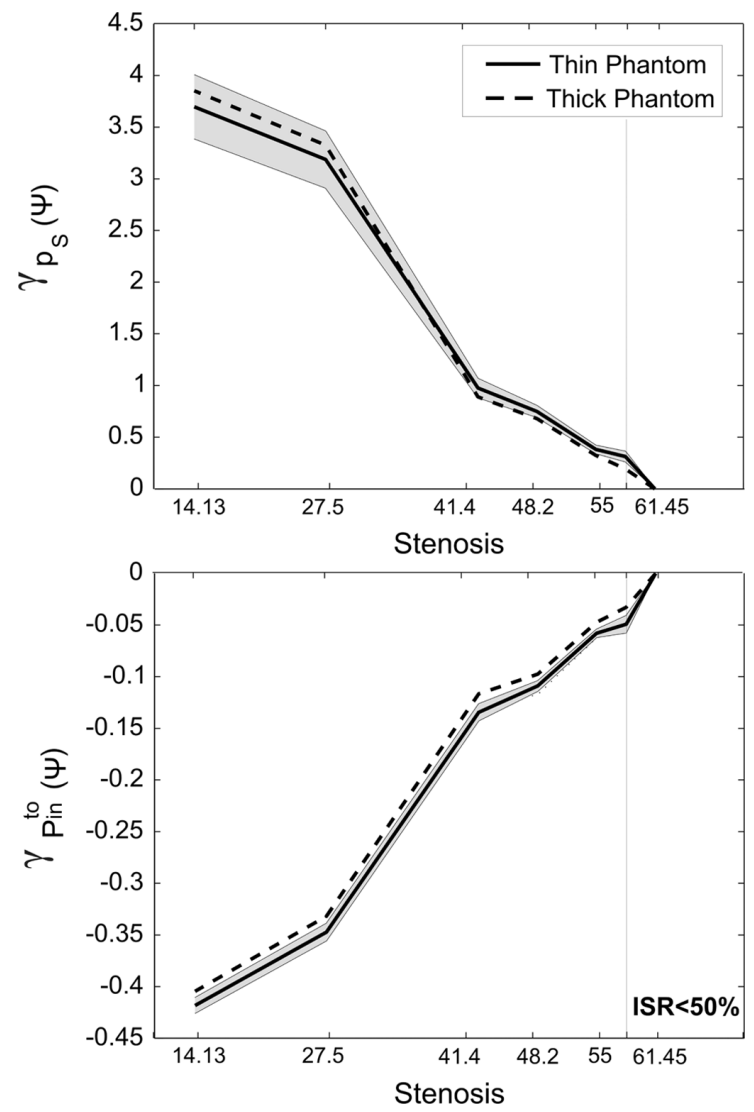

Fig. 12. Normalized scale factor $\gamma$ of a) backscattered power and b) turn on power for the two phantoms, on varying the liquid composition inside the carotid-like pipe. The measured data are sorted according to the real part of the considered complex permittivity.

the possibility to master the sensing and the communication response of the implanted radio sensor by means of the proposed design methodology.

The sensitivity to the tag's status is remarkable even in the early modification of the liquid, corresponding to an $I R S=$ $50 \%$ (from $\bar{\epsilon}_{1}$ to $\bar{\epsilon}_{2}$ ) with a monotonic behavior in the entire considered permittivity range. The turn-on measurement is almost invariant with the observation angle with less than $0.5 \mathrm{~dB}$ uncertainty mainly due to the poor resolution of the reader. An even sharper sensitivity to the vessel's change is finally found by the backscattering analysis, with approximately $5 \mathrm{~dB}$ span between the healthy carotid $\left(\bar{\epsilon}_{1}\right)$ and the full occlusion by plaque $\left(\bar{\epsilon}_{7}\right)$. The measurement variability of the backscattered power is instead not negligible, especially for extremely low values of $\bar{\epsilon}_{\text {stent }}$.

In order to analyze the sensibility of the proposed approach to the variability of the body district, the STENTag has been experimentally characterized also by considering a bigger neck phantom, resembling a stout subject. Such a phantom, referred to as thick phantom, is similar to the one in Fig. 4, except for the different diameter of the large cylinder $(D=13 \mathrm{~cm})$ and for the position of the carotid-like pipe with respect to the border $(p=3$ $\mathrm{cm}$ ). The results (Fig. 11) are similar to those of the previous case except for a $3 \mathrm{~dB}$ scaling since the increased size of the phantom and the deeper position of the radio-sensor produce 
TABLE II

Measured Relative Changes in the STENTAG Responses

\begin{tabular}{|c|c|c|c|c|}
\hline & $\Delta P_{t o}^{i n}(\%)$ & $\Delta P_{R \leftarrow T}(\%)$ & $\Delta \gamma_{P_{t o}^{i n}}(\%)$ & $\Delta \gamma_{p_{S}}(\%)$ \\
\hline \hline $\bar{\epsilon}_{1} \rightarrow \bar{\epsilon}_{2}$ & $11 \%$ & $33 \%$ & $6 \%$ & $38 \%$ \\
\hline $\bar{\epsilon}_{1} \rightarrow \bar{\epsilon}_{7}$ & $56 \%$ & $400 \%$ & $42 \%$ & $370 \%$ \\
\hline
\end{tabular}

a rise of the losses and consequently of the power required to establish the communication.

The uncertainty in the measured data may be however reduced by using all the frequency domain data in Fig. 10 and introducing an averaging operator over frequency. It is hence defined a normalized scale factor:

$$
\gamma_{\xi}=\frac{<\xi(\Psi)-\xi\left(\Psi_{0}\right)>}{<\xi\left(\Psi_{0}\right)>}
$$

where the " $<>$ " brackets indicate the average over frequency samples and $\xi=\left\{P_{\text {in }}^{t o}\right.$ or $\left.p_{s}\right\}$. Fig. 12 shows the averaged $\gamma_{p_{s}}$ and $\gamma_{P_{\mathrm{in}} \text { o }}$ for the two phantoms. For clarity only the standard deviation of the thin case is presented. Since $\gamma$ is a relative parameter, the profiles of the two phantoms are almost overlapped, demonstrating the repeatability of the sensing measurement regardless the human variability. Moreover the use of an averaging indicator sensibly reduces the uncertainty of the data, when compared to Fig. 11. In the worst case the uncertainty is only the $3 \%$ of the mean value, while in the early grade of the restenosis (ISR $<50 \%$ ) it drops to less than $1.5 \%$.

Finally, by a linear fitting of the curves in Figs. 11 and 12, it is possible to estimate the percentage variation of all the previous power indicators referred to the healthy state $\bar{\epsilon}_{0}$ (Table II). In particular, a remarkable $33 \%$ of variation of the STENTag response may be achieved in the early restenosis $\left(\bar{\epsilon}_{1} \rightarrow \bar{\epsilon}_{2}\right)$ by monitoring the changes of the normalized scale factor of the backscattered power and more than 3 times changes at the atherosclerotic restenosis $\left(\bar{\epsilon}_{1} \rightarrow \bar{\epsilon}_{7}\right)$.

\section{CONCLUSION}

Numerical analysis and early experimentation seem to corroborate the possibility to sense some inner biological processes by means of implanted passive RFID tags. These phenomena in evolution produce specific and macroscopic effects on the electromagnetic response of the radio-sensor, which are detectable by the available low-cost technology and with limited power budgets (maximum radiated power less than $1 W$ EIRP), making feasible to embed the required reader capability inside an handheld device and even into a smartphone.

Tag design may benefit from various degrees of freedom: the sensitivity of the radio-sensor can indeed be enhanced by using high impedance ICs, while the inversion curves, relating the expected tag response to the change of the physical phenomena, may be shaped by a wise selection of the impedance matching condition.

The RFID interrogation provides two independent measurements: turn-on power and the backscattered power which, if observed over the frequency, may provide really robust and monotonic metrics, rather immune to the specificity of patients and to the measurement modality. For the considered application to the STENTag problem, the achieved sensing capabilities look enough to fully discriminate the early grade of restenosis $\left(\bar{\epsilon}_{1} \rightarrow \bar{\epsilon}_{2}\right.$ ISR $\left.\simeq 50 \%\right)$ by about a $40 \%$ variation in the measured data.

The described design technique may be however straightforwardly extended to many other implanted metal devices, such as prosthesis, sutures, graft or orthopedic fixings, for which longterm monitoring may provide an added value and turn the patient itself into a primary hub in the emerging personal healthcare systems.

\section{ACKNOWLEDGMENT}

This work was developed under project PRIN-2008: MULTI-TAG funded by Italian Ministry of University. The authors wish to thank Prof. L. De Medici of the Radiology Department of San Camillo Hospital, Roma, for inspiration and discussions.

\section{REFERENCES}

[1] G. Marrocco, L. Mattioni, and C. Calabrese, "Multi-port sensor RFIDs for wireless passive sensing-basic theory and early simulations," IEEE Trans. Antennas Propag, vol. 56, no. 8, pp. 2691-2702, Aug. 2008.

[2] A. Sani, M. Rajab, R. Foster, and Y. Hao, "Antennas and propagation of implanted RFIDs for pervasive healthcare applications," Proc. IEEE, vol. 98 , no. 9, Sep. 2010.

[3] A. Alomainy and Y. Hao, "Modeling and characterization of biotelemetric radio channel from ingested implants considering organ contents," IEEE Trans. Antennas Propag., vol. 57, no. 4, pt. 1, pp. 999-1005, Apr. 2009.

[4] F. Merli, B. Fuchs, J. R. Mosig, and A. K. Skrivervik, "The effect of insulating layers on the performance of implanted antennas," IEEE Trans. Antennas Propag., vol. 58, no. 1, pp. 21-31, 2011.

[5] W. Xia, K. Saito, M. Takahashi, and K. Ito, "Performances of an implanted cavity slot antenna embedded in the human arm," IEEE Trans. Antennas Propag., vol. 57, no. 4, pt. 1, pp. 894-899, Apr. 2009.

[6] [Online]. Available: http://www.positiveidcorp.com/

[7] E. Y. Chow, C.-L. Yang, O. Yuehui, A. L. Chlebowski, P. P. Irazoqui, and W. J. Chappell, "Wireless powering and the study of RF propagation through ocular tissue for development of implantable sensors," IEEE Trans. Antennas Propag., vol. 59, no. 6, pp. 2379-2387, Jun. 2011.

[8] D. Valderas, C. Schmidt, and X. Chen, "Broadband implanted UHF RFID antenna," in Proc. IEEE Antennas and Propagation Society Int. Symp. (APSURSI), Toronto, 2010, pp. 1-4.

[9] H. Rajagopalan and Y. Rahmat-Samii, "Ingestible RFID bio-capsule tag design for medical monitoring," in Proc. IEEE Antennas and Propagation Society Int. Symp. (APSURSI), Toronto, 2010, pp. 1-4.

[10] M. Bitzer, T. Nagele, B. Geist-Barth, U. Klose, E. Gronewaller, M. Morgalla, E. Heiss, and K. Voigt, "Role of hydrodynamic processes in the pathogenesis of peritumoral brain edema in meningioma," $\mathrm{J}$. Neurosug, vol. 39 , no. 4 , pp. $594-604$, Oct. 2000

[11] J. L. Schepps and K. R. Foster, "The UHF and microwave dielectric properties of normal and tumor tissues: Variation in dielectric properties with tissue water content," Phys. Med. Biol., vol. 25, no. 6, pp. $1149-1159,1980$.

[12] A. P. O'Rourke, M. Lazebnik, J. M. Bertram, M. C. Converse, S. C. Hagness, J. G. Webster, and D. M. Mahvi, "Dielectric properties of human normal, malignant and cirrhotic liver tissue: In vivo and ex vivo measurements from 0.5 to $20 \mathrm{GHz}$ using a precision open-ended coaxial probe," Phys. Med. Biol., vol. 52, no. 15, pp. 4707-4719, Aug. 2007.

[13] R. Hoffmann, G. S. Mintz, G. R. Dussaillant, J. J. Popma, A. D. Pichard, L. F. Satler, K. M. Kent, J. Griffin, and M. B. Leon, "Patterns and mechanisms of in-stent restenosis, a serial intravascular ultrasound study," Circulation, vol. 94, pp. 1247-1254, 1996.

[14] M. Habara, M. Terashima, K. Nasu, Y. Kinoshita, E. Tsuchikane, Y. Asakura, O. Kato, and T. Suzuki, "The morphological findings of very late in-stent restenosis after bare-metal stent implant: Optical coherence tomography analysis," Circulation, vol. 122, p. A11302, 2010.

[15] Handbook of Coronary Stents, P. W. Serruys and B. Rensing, Eds., 4th ed. New York: Informa Healthcare, 2001 
[16] P. V. Nikitin and K. V. S. Rao, "Theory and measurement of backscattering from RFID tags," IEEE Antennas Propag. Mag., vol. 48, no. 6, pp. 212-218, Dec. 2006.

[17] G. Marrocco, E. Di Giampaolo, and R. Aliberti, "Estimation of UHF RFID reading regions in real environments," IEEE Antennas Propag. Mag., vol. 51, pp. 44-57, 2010.

[18] G. Marrocco, "The art of UHF RFID antenna design: Impedancematching and size-reduction techniques," IEEE Antennas Propag. Mag., vol. 50, no. 1, pp. 66-96, Feb. 2008.

[19] C. Gabriel and S. Gabriel, "Compilation of the dielectric properties of body tissues at RF and microwave frequencies," [Online]. Available: http://niremf.ifac.cnr.it/docs/DIELECTRIC/home.html

[20] K. V. S. Rao, P. V. Nikitin, and S. F. Lam, "Impedance matching concepts in RFID transponder design," in Proc. 4th IEEE Workshop on Automatic Identification Advanced Technologies, 2005, pp. 39-42.

[21] G. Marrocco, "RFID antennas for the UHF remote monitoring of human subjects," IEEE Tran. Antennas Propag., vol. 55, no. 6, pp. 1862-1870, Jun. 2007.

[22] A. A. El-Menyar, J. A. Suwaidi, and D. R. Holmes, Jr, "Use of drugeluting stents in patients with coronary artery disease and renal insufficiency," Mayo Clinic Proc., vol. 85, no. 2, pp. 165-171, Feb. 2010.

[23] B. K. Lal, E. A. Kaperonis, S. Cuadra, I. Kapadia, and R. W. Hobson II, "Patterns of in-stent restenosis after carotid artery stenting: Classification and implications for long-term outcome," J. Vasc. Surgery, vol. 46 , no. 5, pp. 833-840, Nov. 2007.

[24] Cordis PRECISE Nitinol Stent System [Online]. Available: http://www.cordislabeling.com

[25] E. Y. Chow, Y. Ouyang, B. Beier, W. J. Chappell, and P. P. Irazoqui, "Evaluation of cardiovascular stents as antennas for implantable wireless applications," IEEE Trans. Microw. Theory Tech., vol. 57, no. 10, pt. 2, pp. 2523-2532, Oct. 2009.

[26] L. Sheddena, S. Kennedyb, R. Wadsworthc, and P. Connollyd, "Towards a self-reporting coronary artery stent-measuring neointimal growth associated with in-stent restenosis using electrical impedance techniques," Biosen. Bioelectron., vol. 26, pp. 661-666, 2010.

[27] Boston Scientific Carotid WALLSTENT [Online]. Available: www. bostoscientific.com

[28] Visible Human Project [Online]. Available: http://www.nlm.nih.gov/ research/visible/visible_human.html

[29] G. Hartsgrove, A. Kraszewsky, and A. Surowiec, "Simulated biological materials for electromagnetic radiation absorption studies," Bioelectromagnetics, vol. 8, no. 4, pp. 29-36, 1987.

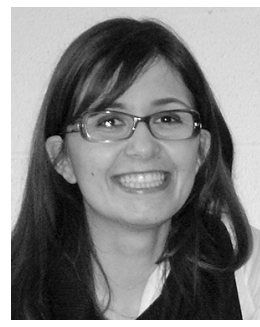

Cecilia Occhiuzzi (M'12) received the M.Sc. degree in medical engineering and the Ph.D. in electromagnetics from the University of Rome "Tor Vergata", in 2008 and 2011, respectively.

Currently, she is a Research Assistant in the University of Rome "Tor Vergata", with interests in wireless health monitoring by means of wearable and implantable radiofrequency identification techniques. In 2008, she was with the School of Engineering, University of Warwick, Warwick, U.K., as a postgraduate student involved with design and implementation of wireless surface acoustic wave (SAW) sensors. In 2010, she was a Visiting Researcher with the Georgia Institute of Technology, Atlanta. Her research was mainly focused on the design of passive RFID sensors for structural health monitoring and gas detection by means of CNT-based tags. She holds two patents on sensor RFID systems.

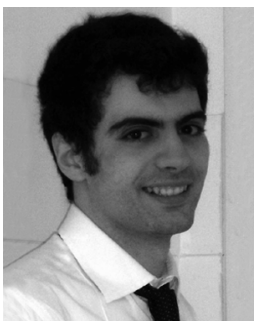

Giordano Contri received the M.Sc. degree in medical engineering from the University of Rome "Tor Vergata", in 2010 and is currently working toward the Ph.D. degree.

His main research interests concern implantable RFID antennas with sensing capabilities. He is working in COSMED (pulmonary function equipment) and is involved in R\&D projects for innovative telemetric devices.

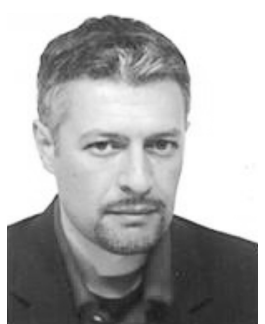

Gaetano Marrocco (M'12) was born on August 19, 1969, in Teramo, Italy. He received the Laurea degree in electronic engineering and the Ph.D. degree in applied electromagnetics from the University of L'Aquila, Italy, in 1994 and 1998, respectively.

$\mathrm{He}$ has been a Researcher at the University of Rome "Tor Vergata" since 1997 where he currently teaches antenna design and bioelectromagnetics. In summer 1994, he was at the University of Illinois at Urbana Champaign, as a Postgraduate Student. In autumn 1999, he was a Visiting Scientist at Imperial College in London. His research is mainly directed to the modelling and design of broadband and ultrawideband antennas and arrays as well as of miniaturized antennas for RFID applications. He has been involved in several space, avionic, naval and vehicular programs of the European Space Agency, NATO, Italian Space Agency, and the Italian Navy. He holds two patents on broadband naval antennas and one patent on sensor RFID systems.

Prof. Marrocco currently serves as an Associate Editor of the IEEE Antennas and Wireless Propagation Letters. 